\title{
Ajustes de quadrado médio do erro em ensaios de competição de cultivares de milho pelo método de Papadakis ${ }^{(1)}$
}

\author{
Alberto Cargnelutti Filho( ${ }^{(2)}$, Lindolfo Storck ${ }^{(3)}$ e Alessandro Dal'Col Lúcio(4)
}

Resumo - O objetivo deste trabalho foi avaliar as diferentes formas de calcular o índice de produtividade, usado como covariável pelo método de Papadakis, e verificar se este método é eficiente para aumentar a precisão experimental nos ensaios de competição de cultivares de milho (Zea mays L.) com grande número de tratamentos. Em relação ao rendimento de grãos, procedeu-se a verificação das pressuposições do modelo matemático, a análise da variância no delineamento de blocos ao acaso e a análise de covariância no delineamento inteiramente casualizado, usando como covariável o índice de produtividade estimado por cinco formas (método de Papadakis). A comparação da análise convencional (blocos ao acaso) com a análise pelo método de Papadakis foi realizada com base nas estimativas do quadrado médio do erro, coeficiente de variação, diferença mínima significativa pelo teste de Tukey, número de grupos formados pelo teste de Scott-Knott e o índice de diferenciação de Fasoulas. O método de Papadakis melhorou a precisão experimental entre 10,5\% e 48,1\% em relação às diferentes formas de estimação da covariável índice de produtividade, mostrando que a vizinhança mais próxima (unidade experimental de referência e as quatro laterais) é a forma mais eficiente na redução do erro experimental.

Termos para indexação: Zea mays, Fasoulas, precisão experimental.

\section{Adjustments of error mean square in corn cultivar competition trials by Papadakis method}

Abstract - The objective of this work was to evaluate the different ways to calculate the yield index, used as covariable for the Papadakis method, and to verify if this method is efficient to increase the experimental precision in corn (Zea mays L.) cultivars competition trials with high number of treatments. The following analysis was performed using grain yield data: mathematical model assumption, analysis of variance in the randomized complete block design (conventional analysis), and analysis of covariance in the completely random design using yield index as a covariable. The yield index covariable was estimated using five different variants of the Papadakis method. The comparisons between the conventional analysis and the Papadakis method was done with the mean square error, coefficient of variation, least significant differences of Tukey test, group numbers of Scott-Knott cluster analysis, and differentiation index of Fasoulas. Papadakis method improved the experimental precision from $10.5 \%$ to $48.1 \%$ in all different ways used to estimate index yield covariable. The four hills neighboring the reference hill was the best index to increase experimental precision.

Index terms: Zea mays, Fasoulas, experimental precision.

\section{Introdução}

Pequenas variações nas unidades experimentais, antes de se aplicar os tratamentos, ou induzidas,

\footnotetext{
(1)Aceito para publicação em 21 de janeiro de 2003. Financiado pelo CNPq

(2)Universidade Federal de Santa Maria (UFSM), PPG Agronomia, CEP 97105-900 Santa Maria, RS. E-mail alberto.filho@net.crea-rs.org.br

(3)UFSM, Dep. de Fitotecnia. Bolsista do CNPq. E-mail: storck@ccr.ufsm.br

(4)UFSM, Dep. de Fitotecnia. E-mail: adlucio@ccr.ufsm.br
}

involuntariamente, durante a execução do experimento, causam heterogeneidade entre as parcelas, também conhecida como variação casual, variação ambiental ou simplesmente erro experimental. A existência do erro na análise dos experimentos requer análise estatística para testar as hipóteses formuladas, que em certos casos, mesmo havendo diferenças entre médias de tratamentos, poderão não ser detectadas pela análise se o erro experimental for grande (Storck et al., 2000a).

Para reduzir o efeito do erro experimental e discriminar melhor as diferenças entre os tratamentos avaliados, deve-se aumentar o número de repetições, 
usar unidades experimentais homogêneas e manejo adequado do ensaio (Storck et al., 2000a). Além disso, se as pressuposições do modelo matemático (aditividade dos efeitos do modelo matemático, e aleatoriedade (independência), homogeneidade das variâncias, normalidade da distribuição dos erros estimados) são satisfeitas, a qualidade da análise de variância é considerada adequada (Marques, 1999). Quando essas pressuposições não são satisfeitas, a análise paramétrica via teste $\mathrm{F}$, as comparações de médias pelos testes de Tukey, Duncan, Scott-Knott, entre outros, ficam prejudicadas e podem levar a falsas conclusões. Logo, deve-se preferir a transformação dos dados em uma nova escala (transformação raiz quadrada, logarítmica, arco seno, entre outras, em função da distribuição dos dados) para que os dados transformados obedeçam, aproximadamente, às pressuposições do modelo matemático ou, então, usar a análise não-paramétrica.

Por causa disso, procura-se minimizar o efeito do erro experimental, buscando fazer inferências precisas, que se deve, a priori, seguir determinadas regras no planejamento e instalação do ensaio, definidas como princípios básicos da experimentação (repetição, casualização e controle local). Além do não uso dos princípios básicos da experimentação e o mau planejamento, fatores como heterogeneidade do material experimental e competições entre e dentro das parcelas aumentam o erro experimental. A presença de ervas daninhas, pragas e doenças e o uso de número insuficiente de amostras também são capazes de afetar o erro experimental (Storck et al., 2000a).

Algumas alternativas de análise espacial, por diversos métodos, têm sido aplicadas objetivando aumentar a precisão experimental (Mendez, 1971; Saez \& Magaña, 1990; Vivaldi, 1990; Zimmerman \& Harville, 1991; Brownie et al., 1993; Amaro \& Cobo, 1994; Stroup et al., 1994; Anoshenko, 1996; Clarke \& Baker, 1996; Amaro et al., 1997; Souza et al., 2000). Esses métodos são estudados há várias décadas e atualmente vêm sendo aplicados com maior freqüência, principalmente pela disponibilidade de técnicas computacionais para a resolução do grande volume de cálculos que exigem. Entre os diversos métodos, o de Papadakis (Papadakis, 1937), baseado na análise de covariância, tem sido alternativa como método de ajuste espacial, o qual utiliza as médias móveis calculadas entre parcelas vizinhas a fim de diminuir o erro em experimentos realizados no campo.

Diversos trabalhos com dados simulados e de experimentos realizados no campo têm demonstrado resultados promissores (Mendez, 1971; Saez \& Magaña, 1990; Vivaldi, 1990; Amaro \& Cobo, 1994; Amaro et al., 1997; Souza et al., 2000). Porém a abrangência espacial das parcelas vizinhas consideradas no cálculo do índice de produtividade não é consenso entre os pesquisadores, haja vista que diferentes formas de cálculo são consideradas (Mendez, 1971; Saez \& Magaña, 1990; Vivaldi, 1990; Brownie et al., 1993; Amaro \& Cobo, 1994; Stroup et al., 1994; Clarke \& Baker, 1996; Amaro et al., 1997; Souza et al., 2000). Os resultados obtidos apontaram para uma tendência de melhoria da precisão com o método de Papadakis, mas não há uniformidade de idéias quanto à magnitude da eficiência desse método, deixando-o numa posição, no mínimo, de igualdade em comparação com outros métodos (Vivaldi, 1990).

A precisão experimental dos ensaios de competição de cultivares de milho é baixa (Lopes \& Storck, 1995; Lúcio, 1997; Marques, 1999), resultando em discriminação ineficiente entre as cultivares.

O objetivo deste trabalho foi estudar as diferentes formas de calcular o índice de produtividade, usado como covariável pelo método de Papadakis, e verificar a eficiência do método no aumento da precisão experimental nos ensaios de competição de cultivares de milho com grande número de tratamentos.

\section{Material e Métodos}

Foram realizados cinco ensaios de competição de cultivares de milho (Zea mays L.) de ciclo precoce, durante os anos agrícolas de 1998/1999, 1999/2000,2000/2001 e 2001/ 2002, no campus da Universidade Federal de Santa Maria, Município de Santa Maria, RS. No ano agrícola 2000/2001, foram realizados dois ensaios, um de alta tecnologia (com irrigação suplementar e três adubações de cobertura) e outro de média tecnologia (sem irrigação e duas adubações de cobertura). Em todos os ensaios, as adubações (base e cobertura) foram realizadas de acordo com análise química do solo, segundo as recomendações de adubação e calagem para os estados do Rio Grande do Sul e Santa Catarina (Comissão de Fertilidade do Solo-RS/SC, 1995). Os tratos 
culturais foram realizados para evitar que a cultura fosse afetada por pragas e doenças e que competisse com plantas daninhas.

O delineamento experimental utilizado foi o de blocos ao acaso, com três repetições, e os blocos espaçados entre si em $1 \mathrm{~m}$. As unidades experimentais constituíram-se de duas fileiras com $5 \mathrm{~m}$ de comprimento espaçadas em $80 \mathrm{~cm}$ (Storck, 1979), sendo a área útil composta pelos $4 \mathrm{~m}$ centrais $\left(6,4 \mathrm{~m}^{2}\right)$. Os tratamentos constituíram-se das cultivares pertencentes à rede de ensaios de competição de cultivares de milho de ciclo precoce, coordenados pela Fepagro. O número de cultivares variou em razão da exclusão e inclusão anual de novas cultivares.

As semeaduras foram realizadas manualmente em 29/10/1998, 21/10/1999, 31/10/2000 e em 9/11/2001, numa profundidade de aproximadamente $5 \mathrm{~cm}$, colocando-se duas sementes/cova; o desbaste de uma plântula em 21/11/1998, $16 / 11 / 1999,15 / 11 / 2000$ e 27/11/2001, respectivamente, ajustou a densidade para 55.000 plantas por hectare.

$\mathrm{Na}$ análise de variância de cada ensaio, foi usada a variável rendimento de grãos ajustada a $13 \%$ de umidade. Foram verificadas as pressuposições do modelo matemático do delineamento blocos ao acaso dos cinco ensaios, conforme Marques (1999).

A seguir, foi realizada a análise de covariância e ajustamento das médias, segundo o delineamento inteiramente casualizado, em que a covariável usada foi o índice de produtividade, estimado pelo método de Papadakis (Papadakis, 1937). Para isto, foi estimado o resíduo de cada unidade experimental, usando a expressão:

$R_{(\mathrm{i}, \mathrm{j})}=\mathrm{Y}_{(\mathrm{i}, \mathrm{j})}-\overline{\mathrm{Y}}_{(\mathrm{i}, \mathrm{.})}$,

em que: $\mathrm{R}_{(\mathrm{i}, \mathrm{j})}$ é o resíduo da observação com o i-ésimo tratamento e j-ésima repetição; $\mathrm{Y}_{(\mathrm{i}, \mathrm{j})}$ é o valor observado na unidade experimental com o i-ésimo tratamento e j-ésima repetição e $\overline{\mathrm{Y}}_{(\mathrm{i},)}$ é a média das unidades experimentais com o i-ésimo tratamento.

Os valores $\mathrm{R}_{(\mathrm{i}, \mathrm{j})}$ foram posicionados segundo a casualização de cada um dos cinco ensaios, recebendo a notação $\mathrm{R}_{(\mathrm{m}, \mathrm{n})}$, para $\mathrm{m}=1,2,3$ blocos (filas) e $\mathrm{n}=1,2,3$, $\ldots, \mathrm{n}$ cultivares (colunas). Assim, $\mathrm{R}_{(1,2)}$ refere-se ao resíduo da parcela posicionada no bloco 1 e coluna 2 . $O$ índice de produtividade $(\mathrm{F})$ foi calculado, em cada unidade experimental, de cinco formas, com variações nas tendências geográficas (abrangências variam quanto à posição e quanto ao número, o que resulta em variação na eficiência da covariável), isto é, $\mathrm{F}_{\mathrm{k}(\mathrm{m}, \mathrm{n})}, \mathrm{k}=1,2, \ldots, 5$ formas:

$\mathrm{F}_{1(\mathrm{~m}, \mathrm{n})}=\left[\mathrm{R}_{(\mathrm{m}, \mathrm{n})}+\mathrm{R}_{(\mathrm{m}, \mathrm{n}-1)}+\mathrm{R}_{(\mathrm{m}, \mathrm{n}+1)}+\mathrm{R}_{(\mathrm{m}-1, \mathrm{n})}+\mathrm{R}_{(\mathrm{m}+1, \mathrm{n})}\right] / 5$;

$\mathrm{F}_{2(\mathrm{~m}, \mathrm{n})}=\left[\mathrm{R}_{(\mathrm{m}, \mathrm{n})}+\mathrm{R}_{(\mathrm{m}, \mathrm{n}-2)}+\mathrm{R}_{(\mathrm{m}, \mathrm{n}-1)}+\mathrm{R}_{(\mathrm{m}, \mathrm{n}+1)}+\mathrm{R}_{(\mathrm{m}, \mathrm{n}+2)}\right] / 5$;

$\mathrm{F}_{3(\mathrm{~m}, \mathrm{n})}=\left[5 \mathrm{~F}_{1(\mathrm{~m}, \mathrm{n})}+\mathrm{R}_{(\mathrm{m}, \mathrm{n}-2)}+\mathrm{R}_{(\mathrm{m}, \mathrm{n}+2)}\right] / 7$;

$\mathrm{F}_{4(\mathrm{~m}, \mathrm{n})}=\left[7 \mathrm{~F}_{3(\mathrm{~m}, \mathrm{n})}+\mathrm{R}_{(\mathrm{m}-1, \mathrm{n}-1)}+\mathrm{R}_{(\mathrm{m}-1, \mathrm{n}+1)}+\mathrm{R}_{(\mathrm{m}+1, \mathrm{n}-1)}+\right.$
$\left.\mathrm{R}_{(\mathrm{m}+1, \mathrm{n}+1)}\right] / 11$;

$\mathrm{F}_{5(\mathrm{~m}, \mathrm{n})}=\left[11 \mathrm{~F}_{4(\mathrm{~m}, \mathrm{n})}+\mathrm{R}_{(\mathrm{m}-1, \mathrm{n}-2)}+\mathrm{R}_{(\mathrm{m}-1, \mathrm{n}+2)}+\mathrm{R}_{(\mathrm{m}+1, \mathrm{n}-2)}+\right.$ $\left.\mathrm{R}_{(\mathrm{m}+1, \mathrm{n}+2)}\right] / 15$.

Nos casos em que a posição $(m ; n)$ recai nas extremidades, o valor do índice $\mathrm{F}_{\mathrm{k}(\mathrm{m}, \mathrm{n})}$ é obtido pela média dos $\mathrm{R}_{(\mathrm{m}, \mathrm{n})}$ disponíveis.

A avaliação da precisão experimental nas análises segundo o delineamento blocos ao acaso e na análise de covariância, nas cinco formas de estimativa do índice de produtividade (método de Papadakis), foi procedida com base nas estimativas das estatísticas: quadrado médio do erro $\left(\mathrm{QM}_{\mathrm{E}}\right)$; coeficiente de variação $(\mathrm{CV})$; diferença mínima significativa entre as cultivares pelo teste de Tukey $(\Delta)$ [em que: $\Delta=\mathrm{q}_{\mathrm{o}\left(\mathrm{n} ; \mathrm{GL}_{\mathrm{E}}\right)} \sqrt{\mathrm{QM}_{\mathrm{E}} / \mathrm{J}} ; \mathrm{q}_{\mathrm{\alpha (n}, \mathrm{GL}}$ é o valor da Tabela para o teste de Tukey; $n$ é o número de cultivares; $\mathrm{GL}_{\mathrm{E}}$ é o grau de liberdade do erro; $\mathrm{QM}_{\mathrm{E}}$ é a estimativa do erro experimental; J é o número de repetições]; diferença mínima significativa entre as cultivares pelo teste de Tukey em porcentagem da média (DMS) [em que: DMS $=100 * N \hat{\mathrm{m}} ; \hat{\mathrm{m}}$ é a estimativa da média geral do ensaio]; estatística HD (Storck et al., 2000b) cuja estimativa é obtida dividindo-se a amplitude das médias dos tratamentos pelo valor do $\Delta$ de Tukey; número de grupos formados pelo teste de Scott-Knott (Scott \& Knott, 1974); e índice de diferenciação (D) de Fasoulas (Fasoulas, 1983), o qual é estimado pela expressão $D=200 \sum \mathrm{m} /[\mathrm{n}(\mathrm{n}-1)]$, sendo $\mathrm{n}$ o número total de cultivares e $\mathrm{m}$ o número de médias que uma determinada cultivar supera estatisticamente, após a aplicação do teste de Tukey, fornecendo o porcentual de diferenças estatísticas entre médias que o método de comparação múltiplas de médias (Tukey) consegue detectar.

As análises e obtenção das estimativas das estatísticas foram realizadas por meio dos programas computacionais NTIA/Embrapa, SAEG/UFV e pelo aplicativo Office Excel.

\section{Resultados e Discussão}

Em nenhum dos cinco ensaios, nos quatro anos agrícolas, houve violação das pressuposições do modelo matemático em relação à variável rendimento de grãos, comprovando que os testes de hipóteses paramétricos são adequados. A análise da variância da variável índice de produtividade mostrou efeito não-significativo em todos os ensaios e formas, indicando que esta variável não é influenciada pelos tratamentos (cultivares).

A variância entre blocos foi significativa (blocos heterogêneos), em relação ao rendimento de grãos de milho em dois ensaios, indicando que o uso de 
blocos nos ensaios não pode ser descartado (Tabela 1). Por sua vez, o efeito de cultivares, na análise da variância em blocos ao acaso, foi significativo em quatro ensaios. Resultados semelhantes foram obtidos por Marques (1999), em que 47,56\% dos ensaios tiveram efeito significativo de blocos e $85,67 \%$ efeito significativo de cultivares. Quando os dados foram analisados pelo método de Papadakis, o efeito de cultivar foi significativo nos cinco ensaios, independentemente da forma de cálculo do índice de produtividade usado como covariável, mostrando melhor discriminação entre as cultivares.

O efeito da covariável índice de produtividade, usada no método de Papadakis, foi significativo em todos os ensaios e formas, indicando que as médias das cultivares podem ser ajustadas pela covariável e que existe eficiência no método (Tabela 1).
As diferentes formas de cálculo do índice de produtividade mostraram que o número e a localização das parcelas vizinhas interferem na eficiência do método de Papadakis (Tabela 1). Isto pode ser explicado porque, até uma determinada distância, os dados possuem maior dependência, a partir da qual passam a ser independentes de acordo com as características físicas, químicas e biológicas do solo. Com exceção do ensaio realizado no ano agrícola 2000/2001, usando média tecnologia (Papadakis forma 5), o método de Papadakis reduziu o quadrado médio do erro $\left(\mathrm{QM}_{\mathrm{E}}\right)$, independentemente da forma utilizada no cálculo do índice de produtividade. A redução variou de 2,9\% (ano agrícola 1999/2000 forma 5) a 57,4\% (ano agrícola 2000/2001, com média tecnologia e cálculo do índice de produtividade na forma 1). Na média dos cinco ensaios, a redução va-

Tabela 1. Número de graus de liberdade (GL) e estimativas de quadrados médios (QM) das causas de variação da análise da variância segundo o delineamento blocos ao acaso e a análise de covariância pelo método de Papadakis, nas diferentes formas de estimativa da covariável índice de produtividade (Índice), e porcentagem de redução do quadrado médio do erro (Redução) pelo método de Papadakis, nas diferentes formas e ensaios de milho de ciclo precoce. Santa Maria, RS, 2002.

\begin{tabular}{|c|c|c|c|c|c|c|c|c|c|c|}
\hline \multirow{2}{*}{$\begin{array}{l}\text { Causas de } \\
\text { variação }\end{array}$} & \multicolumn{2}{|c|}{$1998 / 1999$} & \multicolumn{2}{|c|}{$1999 / 2000$} & \multicolumn{2}{|c|}{$2000 / 2001^{(1)}$} & \multicolumn{2}{|c|}{$2000 / 2001^{(2)}$} & \multicolumn{2}{|c|}{$2001 / 2002$} \\
\hline & GL & QM & GL & $\overline{\mathrm{QM}}$ & GL & QM & GL & $\overline{\mathrm{QM}}$ & GL & QM \\
\hline & \multicolumn{10}{|c|}{ Delineamento blocos ao acaso } \\
\hline Bloco & 2 & $4,764 *$ & 2 & $1,992^{\mathrm{ns}}$ & 2 & $1,559^{\mathrm{ns}}$ & 2 & $8,255^{*}$ & 2 & $0,299^{\mathrm{ns}}$ \\
\hline Cultivar & 29 & $1,619^{*}$ & 22 & $2,768 *$ & 29 & $2,435 *$ & 29 & $1,644^{*}$ & 29 & $1,306^{\mathrm{ns}}$ \\
\hline \multirow[t]{2}{*}{ Erro } & 58 & 0,408 & 44 & 0,798 & 58 & 0,708 & 58 & 0,815 & 58 & 0,830 \\
\hline & \multicolumn{10}{|c|}{ Papadakis: forma 1} \\
\hline Cultivar & 29 & $1,434 *$ & 22 & $2,889^{*}$ & 29 & $2,159 *$ & 29 & $1,915^{*}$ & 29 & $1,481^{*}$ \\
\hline Índice & 1 & $19,812 *$ & 1 & $16,259 *$ & 1 & $25,648 *$ & 1 & $43,290^{*}$ & 1 & $22,451 *$ \\
\hline Erro & 59 & 0,227 & 45 & 0,507 & 59 & 0,314 & 59 & 0,347 & 59 & 0,446 \\
\hline \multirow[t]{2}{*}{ Redução (\%) } & & 44,4 & & 36,5 & & 55,8 & & 57,4 & & 46,3 \\
\hline & \multicolumn{10}{|c|}{ Papadakis: forma 2} \\
\hline Cultivar & 29 & $1,548 *$ & 22 & $3,019 *$ & 29 & $2,218^{*}$ & 29 & $1,593 *$ & 29 & $1,310^{*}$ \\
\hline Índice & 1 & $19,671 *$ & 1 & $12,019 *$ & 1 & $21,816^{*}$ & 1 & $36,776^{*}$ & 1 & $19,392 *$ \\
\hline Erro & 59 & 0,229 & 45 & 0,602 & 59 & 0,379 & 59 & 0,458 & 59 & 0,497 \\
\hline \multirow[t]{2}{*}{ Redução (\%) } & & 43,9 & & 24,6 & & 46,5 & & 43,8 & & 40,1 \\
\hline & \multicolumn{10}{|c|}{ Papadakis: forma 3} \\
\hline Cultivar & 29 & $1,410 *$ & 22 & $2,993^{*}$ & 29 & $2,118^{*}$ & 29 & $1,838^{*}$ & 29 & $1,396^{*}$ \\
\hline Índice & 1 & $18,986^{*}$ & 1 & $13,049^{*}$ & 1 & $21,827 *$ & 1 & $39,794 *$ & 1 & $20,530 *$ \\
\hline Erro & 59 & 0,241 & 45 & 0,579 & 59 & 0,379 & 59 & 0,407 & 59 & 0,478 \\
\hline \multirow[t]{2}{*}{ Redução (\%) } & & 40,9 & & 27,4 & & 46,5 & & 50,1 & & 42,4 \\
\hline & \multicolumn{10}{|c|}{ Papadakis: forma 4} \\
\hline Cultivar & 29 & $1,350 *$ & 22 & $2,899 *$ & 29 & $2,157^{*}$ & 29 & $1,886^{*}$ & 29 & $1,497 *$ \\
\hline Índice & 1 & $15,752 *$ & 1 & $11,732 *$ & 1 & $17,160 *$ & 1 & $22,936^{*}$ & 1 & $17,012 *$ \\
\hline Erro & 59 & 0,295 & 45 & 0,608 & 59 & 0,458 & 59 & 0,692 & 59 & $0,538^{*}$ \\
\hline \multirow[t]{2}{*}{ Redução (\%) } & & 27,7 & & 23,8 & & 35,3 & & 15,1 & & 35,2 \\
\hline & \multicolumn{10}{|c|}{ Papadakis: forma 5} \\
\hline Cultivar & 29 & $1,383^{*}$ & 22 & $2,705^{*}$ & 29 & $2,165^{*}$ & 29 & $1,774^{*}$ & 29 & $1,442 *$ \\
\hline Índice & 1 & $11,086^{*}$ & 1 & $4,233^{*}$ & 1 & $12,845^{*}$ & 1 & $12,736^{*}$ & 1 & $10,739 *$ \\
\hline Erro & 59 & 0,374 & 45 & 0,775 & 59 & 0,531 & 59 & 0,865 & 59 & 0,644 \\
\hline Redução (\%) & & 8,3 & & 2,9 & & 25,0 & & $-6,1$ & & 22,4 \\
\hline
\end{tabular}

(1) Alta tecnologia. ${ }^{(2)}$ Média tecnologia. nsNão-significativo. * Significativo a $5 \%$ de probabilidade pelo teste $\mathrm{F}$. 
riou de $10,5 \%$ (forma 5 ) a 48,1\% (forma 1), podendose inferir que a forma 1 foi a mais eficiente na redução do erro experimental. Resultados semelhantes foram encontrados por Saez \& Magaña (1990), que, ao analisarem 30 ensaios pelo método de Papadakis, cuja estimativa do índice de produtividade foi obtida conforme a forma 1, obtiveram redução média de $55,11 \%$ no erro experimental.

As formas 2 e 3 apresentaram reduções intermediárias e semelhantes entre si. A menor distância entre as parcelas vizinhas utilizadas no cálculo da forma 2 pode torná-las mais dependentes e, como conseqüência, mais homogêneas. Logo, a análise de covariância com o índice de produtividade pelo método de Papadakis não diminui muito o $\mathrm{QM}_{\mathrm{E}} \mathrm{e} \mathrm{o}$ envolvimento de parcelas mais distantes na forma 1 , provavelmente mais heterogêneas entre si, o que pode explicar a maior redução do $\mathrm{QM}_{\mathrm{E}}$. Já nas formas 4 e 5 , apesar do uso de parcelas mais distantes em relação à referência, o maior número de parcelas vizinhas envolvidas na estimativa do índice de produtividade fez com que a média se aproximasse da média da área total do ensaio, podendo explicar a menor eficiência. Esses resultados classificam as formas 4 e 5 como as de baixa eficiência; 2 e 3, as de média eficiência; e a 1 como a de alta eficiência em reduzir o erro experimental.
Nas três melhores formas (1, 2 e 3), a menor redução do $\mathrm{QM}_{\mathrm{E}}$ foi obtida no ensaio do ano agrícola 1999/2000, pois com um menor número de cultivares houve maior homogeneidade entre as unidades experimentais dentro do bloco, podendo este ser um dos fatores que explica a menor redução do $\mathrm{QM}_{\mathrm{E}}$ (Tabela 1). Assim, quanto maior o número de tratamentos, maior é a eficiência do método de Papadakis (Amaro \& Cobo, 1994).

Com exceção do número de grupos formados pelo teste de Scott-Knott nos ensaios dos anos agrícolas 1999/2000 e 2000/2001, usando alta tecnologia, todas as estatísticas utilizadas na comparação da precisão obtida pelo método de Papadakis (forma 1) em relação à análise em blocos ao acaso indicaram melhor eficiência desse método, ou seja, o $\mathrm{QM}_{\mathrm{E}}, \mathrm{CV}, \Delta$ e a DMS foram menores e as estatísticas HD, SK e D foram maiores, revelando melhoria na discriminação das cultivares (Tabela 2).

Conforme classificação de Lúcio et al. (1999), ensaios de cultivares de milho com DMS entre $9,1 \%$ a $25,5 \%$ e $25,5 \%$ a $48,4 \%$ são classificados como de alta e média precisão experimental, respectivamente. Assim, de acordo com os resultados da DMS, os cinco ensaios são de média precisão (Tabela 2). Esta precisão experimental está próxima da relatada por Lopes \& Storck (1995) e Marques (1999). Por sua vez, quando os dados foram analisados pelo método

Tabela 2. Estatísticas da análise da variância segundo o delineamento blocos ao acaso (DBA) e análise da covariância pelo método de Papadakis com a estimativa do índice de produtividade obtido pela forma 1 (PAP) nos cinco ensaios de milho de ciclo precoce. Santa Maria, RS, 2002.

\begin{tabular}{|c|c|c|c|c|c|c|c|c|c|c|}
\hline \multirow[t]{2}{*}{ Estatísticas $^{(1)}$} & \multicolumn{2}{|c|}{ 1998/1999 } & \multicolumn{2}{|c|}{$1999 / 2000$} & \multicolumn{2}{|c|}{$2000 / 2001^{(2)}$} & \multicolumn{2}{|c|}{$2000 / 2001^{(3)}$} & \multicolumn{2}{|c|}{$2001 / 2002$} \\
\hline & DBA & PAP & DBA & PAP & DBA & PAP & DBA & PAP & DBA & PAP \\
\hline $\mathrm{QM}_{\mathrm{E}}$ & 0,408 & 0,227 & 0,798 & 0,507 & 0,708 & 0,314 & 0,815 & 0,347 & 0,830 & 0,446 \\
\hline $\mathrm{GL}_{\mathrm{E}}$ & 58 & 59 & 44 & 45 & 58 & 59 & 58 & 59 & 58 & 59 \\
\hline I & 30 & 30 & 23 & 23 & 30 & 30 & 30 & 30 & 30 & 30 \\
\hline $\mathrm{J}$ & 3 & 3 & 3 & 3 & 3 & 3 & 3 & 3 & 3 & 3 \\
\hline Média & 5,176 & 5,176 & 6,088 & 6,088 & 7,243 & 7,243 & 6,134 & 6,134 & 6,234 & 6,234 \\
\hline $\mathrm{CV}(\%)$ & 12,34 & 9,20 & 14,67 & 11,70 & 11,62 & 7,74 & 14,72 & 9,61 & 14,61 & 10,71 \\
\hline$\Delta$ & 2,054 & 1,532 & 2,837 & 2,261 & 2,706 & 1,802 & 2,903 & 1,894 & 2,930 & 2,148 \\
\hline $100 \Delta$ /média & 40,0 & 29,6 & 46,6 & 37,1 & 37,4 & 24,9 & 47,3 & 30,9 & 47,0 & 34,5 \\
\hline Amplitude & 2,383 & 2,586 & 3,544 & 3,554 & 3,531 & 3,404 & 2,647 & 3,203 & 3,051 & 3,245 \\
\hline HD & 1,16 & 1,69 & 1,25 & 1,57 & 1,30 & 1,90 & 0,91 & 1,70 & 1,04 & 1,51 \\
\hline SK & 2 & 4 & 2 & 2 & 3 & 3 & 2 & 3 & 1 & 2 \\
\hline D & 3,9 & 11,5 & 1,6 & 11,5 & 3,9 & 12,4 & 0 & 9,9 & 0,2 & 3,0 \\
\hline
\end{tabular}

${ }^{(1)} \mathrm{QM}_{\mathrm{E}}$ : quadrado médio do erro; GLE: número de graus de liberdade do erro; I: número de cultivares; J: número de repetições; Média: média do rendimento de grãos das cultivares de milho em t/ha; $C V(\%)$ : coeficiente de variação; $\Delta$ : diferença mínima significativa pelo teste de Tukey em toneladas; 100 /média: diferença mínima significativa pelo teste de Tukey em porcentagem da média; HD: amplitude entre as médias de cultivares, amplitude/ $\Delta$; SK: número de grupos formados pelo teste de Scott-Knott em nível de 5\% de probabilidade de erro; D: índice de diferenciação de Fasoulas. ${ }^{(2)}$ Alta tecnologia. ${ }^{(3)}$ Média tecnologia 
de Papadakis, ano agrícola 2000/2001, com alta tecnologia, a precisão experimental passou para alta. Nos demais ensaios permaneceu com precisão média, com valores da DMS inferiores aos obtidos pela análise convencional. Na média, a DMS passou de $43,66 \%$, quando analisada em blocos ao acaso, para $31,40 \%$, pelo método de Papadakis.

O índice de diferenciação de Fasoulas (D) do delineamento blocos ao acaso foi menor que o obtido pelo método de Papadakis, nos cinco ensaios (Tabela 2). Nos quatro ensaios com 30 cultivares e no ensaio com 23 cultivares, o número de pares de contrastes possíveis é 435 e 253 , respectivamente; o número de contrastes significativos no delineamento blocos ao acaso, nos cinco ensaios, variou de zero a 17, enquanto o método de Papadakis detectou de 13 a 54 pares de comparações com diferenças significativas.

Uma vez que a metodologia não constitui consenso entre os pesquisadores, recomenda-se maior cuidado no planejamento dos experimentos, quanto à definição dos objetivos da pesquisa, à escolha do delineamento experimental apropriado, ao tamanho ideal da unidade experimental e a outras técnicas experimentais, como a eventual necessidade de bordadura, entre outras.

\section{Conclusões}

1. O método de Papadakis é alternativa, em casos especiais, de redução do erro experimental para melhorar a discriminação entre as cultivares.

2. A estimativa do índice de produtividade da parcela de referência e das quatro laterais mais próximas é eficiente na redução do erro experimental.

\section{Referências}

AMARO, R.; COBO, M. La investigación agrícola y la aplicación del método de Papadakis. Revista de la Facultad de Agronomia, Macaray, v. 20, n. 1, p. 35-45, 1994.

AMARO, R.; COBO, M.; QUIROZ, A. Comparación de metodos de ajuste en el analisis espacial de experimentos de campo. Agronomia Tropical, Macaray, v. 47, n. 3, p. 359-374, 1997.

ANOSHENKO, B. Y. Local adjustment method for field experiments-1: the method and its examination by computer simulation. Euphytica, Dordrecht, v. 90, n. 2, p. 137-148, 1996.

BROWNIE, C.; BOWMAN, D. T.; BURTON, J. W. Estimating spatial variation in analysis of data from yield trials: a comparison of methods. Agronomy Journal, Madison, v. 85, p. 1244-1253, 1993.

CLARKE, F. R.; BAKER, R. J. Spatial analysis improves precision of seed lot comparisons. Crop Science, Madison, v. 36, p. 1180-1184, 1996.

COMISSÃO DE FERTILIDADE DO SOLO-RS/SC (Passo Fundo, RS). Recomendações de adubação e calagem para o Estado do Rio Grande do Sul e Santa Catarina. 3. ed. Passo Fundo: Sociedade Brasileira de Ciência do Solo/ Embrapa-CNPT, 1995. 223 p.

FASOULAS, A. C. Rating cultivars and trials in applied plant breeding. Euphytica, Dordrecht, v. 32, n. 3, p. 939-943, 1983.

LOPES, S. J.; STORCK, L. A precisão experimental para diferentes manejos na cultura do milho. Ciência Rural, Santa Maria, v. 25, n. 1, p. 49-53, 1995.

LÚCIO, A. D. Parâmetros da precisão experimental das principais culturas anuais do Estado do Rio Grande do Sul. 1997. 64 f. Dissertação (Mestrado em Agronomia) - Universidade Federal de Santa Maria, Santa Maria, 1997.

LÚCIO, A. D.; STORCK, L.; BANZATTO, D. A. Classificação dos experimentos de competição de cultivares quanto a sua precisão. Pesquisa Agropecuária Gaúcha, Porto Alegre, v. 5, n. 1, p. 99-103, 1999.

MARQUES, D. G. As pressuposições e a precisão dos ensaios de competição de cultivares de milho no Estado do Rio Grande do Sul. 1999. 42 f. Dissertação (Mestrado em Agronomia) - Universidade Federal de Santa Maria, Santa Maria, 1999.

MENDEZ, I. Estudio de seis metodos alternativos para el uso de bloques en la experimentación de campo. Agrociencia, Chapingo, n. 6, p. 3-16, 1971.

PAPADAKIS, J. S. Méthode statistique pour des expériences sur champ. Thessalonike: Institut d'Amélioration des Plantes à Salonique, 1937. 30 p. (Bulletin, 23).

SAEZ, T. C.; MAGAÑA, J. A. J. El análisis de experimentos por método Papadakis. Chapingo, Chapingo, v. 15, n. 71/72, p. 110-113, 1990.

SCOTT, A. J.; KNOTT, M. A. A cluster analysis method for grouping means in the analysis of variance. Biometrics, Raleigh, v. 30, n. 3, p. 507-512, 1974. 
SOUZA, E. A.; GERALDI, I. O.; RAMALHO, M. A. P. Alternativas experimentais na avaliação de famílias em programas de melhoramento genético de feijoeiro. Pesquisa Agropecuária Brasileira, Brasília, v. 35, n. 9, p. $1765-1771$, set. 2000 .

STORCK, L. Estimativa para tamanho e forma de parcela e número de repetições para experimentos com milho (Zea mays L.). 1979. 98 f. Dissertação (Mestrado em Agronomia) - Universidade Federal do Rio Grande do Sul, Porto Alegre, 1979.

STORCK, L.; GARCIA, D. C.; LOPES, S. J.; ESTEFANEL, V. Experimentação vegetal. Santa Maria: UFSM, 2000a. 198 p.

STORCK, L.; LOPES, S. J.; MARQUES, D. G.; TISOTT, C. A.; ROS, C. A. Análise de covariância para melhoria da capacidade de discriminação em ensaios de cultivares de milho. Pesquisa Agropecuária Brasileira, Brasília, v. 35, n. 7, p. 1311-1316, jul. 2000b.

STROUP, W. W.; BAENZIGER, P. S.; MULITZE, D. K. Removing spatial variation from wheat yield trials: a comparison of methods. Crop Science, Madison, v. 86, p. 62-66, 1994.

VIVALDI, L. J. Comparação entre métodos de análise espacial de experimentos de campo. Pesquisa Agropecuária Brasileira, Brasília, v. 25, n. 1, p. 77-84, 1990.

ZIMMERMAN, D. L.; HARVILLE, D. A. A random field approach to the analysis of field-plot experiments and other spatial experiments. Biometrics, Washington, v. 47, p. 223-239, 1991 